\title{
Measurement of the Solar Absorptance and Thermal Emittance of Lunar Simulants
}

\author{
James R. Gaier ${ }^{1}$, Kenneth W. Street ${ }^{2}$ \\ NASA Glenn Research Center, Cleveland, OH 44135 \\ and \\ Robert J. Gustafson ${ }^{3}$ \\ Orbital Technologies, Madison, WI 53717
}

\begin{abstract}
The first comparative study of the reflectance spectra of lunar simulants is presented. All of the simulants except one had a wavelength-dependant reflectivity, $\rho(\lambda)$, near 0.10 over the wavelength range of 8 to $25 \mu \mathrm{m}$, so they are highly emitting at room temperature and lower. The $300 \mathrm{~K}$ emittance, $\varepsilon$, of all the lunar simulants except one ranged from 0.884 to 0.906 . The $300 \mathrm{~K} \varepsilon$ of JSC Mars-1 simulant was 0.927. There was considerably more variation in the lunar simulant reflectance in the solar spectral range $(250$ to $2500 \mathrm{~nm})$ than in the thermal infrared. Larger particle size simulants reflected much less than those with smaller particle size. As expected, the lunar highlands simulants were more reflective in this wavelength range than the lunar mare simulants. The $\alpha$ of the simulants ranged from 0.413 to 0.817 for those with smaller particles and 0.669 to 0.906 for large particles. Although spectral differences were observed, the total integrated $\alpha$ for the simulants appears to be similar to that of lunar soils $(0.65$ to 0.88$)$. These data are now available to be used in modeling the effects of dust on thermal control surfaces.
\end{abstract}

\section{Nomenclature}

$\begin{array}{ll}\mathrm{AM} 0 & =\text { air mass zero, the solar spectrum at } 1 \mathrm{AU} \text { without air absorption } \\ \mathrm{R} & =\text { residual difference between the experimental and calculated data } \\ \mathrm{T}(\lambda) & =\text { wavelength-dependent transmission } \\ \alpha & =\text { total integrated hemispherical absorptance over the solar spectrum }(250 \text { to } 2500 \mathrm{~nm}) \\ \alpha(\lambda) & =\text { wavelength-dependant absorptivity } \\ \varepsilon & =\text { total integrated hemispherical emittance over thermal range (100 to } 400 \mathrm{~K}) \\ \rho & =\text { total integrated hemispherical reflectance } \\ \rho(\lambda) & =\text { wavelength-dependant reflectivity }\end{array}$

\section{Introduction}

\begin{abstract}
s NASA prepares to return humans to the moon, extensive efforts are under way to design the thermal control systems that will protect the Altair lunar lander and the lunar surface system elements from the harsh lunar thermal environment. Part of this effort is to determine the effects of dust deposited on the thermal control surfaces. But even a casual glance up at the moon reveals that the lunar surface is not uniform in brightness. The albedo of specific areas of the moon varies from 0.50 for the brightest features to 0.07 for the darkest. ${ }^{1}$ If the dust is darker, then more light will be absorbed by it, resulting in increased heating both of the dust and, through conduction and

\footnotetext{
${ }^{1}$ Research Physicist, Space Environment and Experiments Branch, MS 309-2, NASA Glenn Research Center, 21000 Brookpark Road, Cleveland OH 44135.

${ }^{2}$ Materials Research Engineer, Tribology and Mechanical Components Branch, MS 23-2, NASA Glenn Research Center, 21000 Brookpark Road, Cleveland OH 44135.

${ }^{3}$ Principal Aerospace Engineer, Orbital Technologies Corporation, Space Center, 1212 Fourier Drive, Madison, WI 53717.
} 
radiation, any thermal control surface it may land on. To quantify these effects, lunar simulants must be developed that have a variety of hues. But the albedo is typically measured in the visible region (400 to $700 \mathrm{~nm}$ wavelength), and the solar radiation which heats thermal control surfaces is intense over a wider region, 250 to $2500 \mathrm{~nm}$ (Fig. 1). Further, the reflectance $(\rho)$ is actually a more useful quantity than albedo, since it is a material property derived from the conservation law:

$$
\alpha(\lambda)+\rho(\lambda)+T(\lambda)=1
$$

where $T(\lambda), \alpha(\lambda)$ and $\rho(\lambda)$ are the spectral transmittance of light through the material, the spectral absorptivity, and spectral reflectivity, respectively. Under the condition where $T=0, \alpha(\lambda)$ is given by $[1-\rho(\lambda)]$ and through Kirchhoff's Law, $\alpha(\lambda)=\varepsilon(\lambda)$, the spectral emittance, for the same temperature and wavelength. The $\rho(\lambda)$ of a variety of lunar simulants was measured over the 250 to $2500 \mathrm{~nm}$ range. This was convoluted with the air mass zero (AM0) solar spectrum and the integrated absorptance $(\alpha=[1-\rho]$ for opaque surfaces) was calculated for each simulant. In addition to heating the thermal control surfaces through absorption, the dust may also affect the ability of the thermal control surface to radiate heat in the thermal infrared. The relationship between wavelength and temperature can be approximated by the Raleigh-Jeans law, which determines the wavelength at which the intensity for a blackbody radiation curve at a given temperature is maximum. Temperatures of interest and their corresponding wavelengths are shown in Table I. In this study, the integrated emittance $(\varepsilon)$ of the simulants over the 2 to $25 \mu \mathrm{m}$ wavelength range, corresponding to temperatures as low as $115 \mathrm{~K}$, was calculated as well as the $\alpha$ over the 250 to $2500 \mathrm{~nm}$ range.

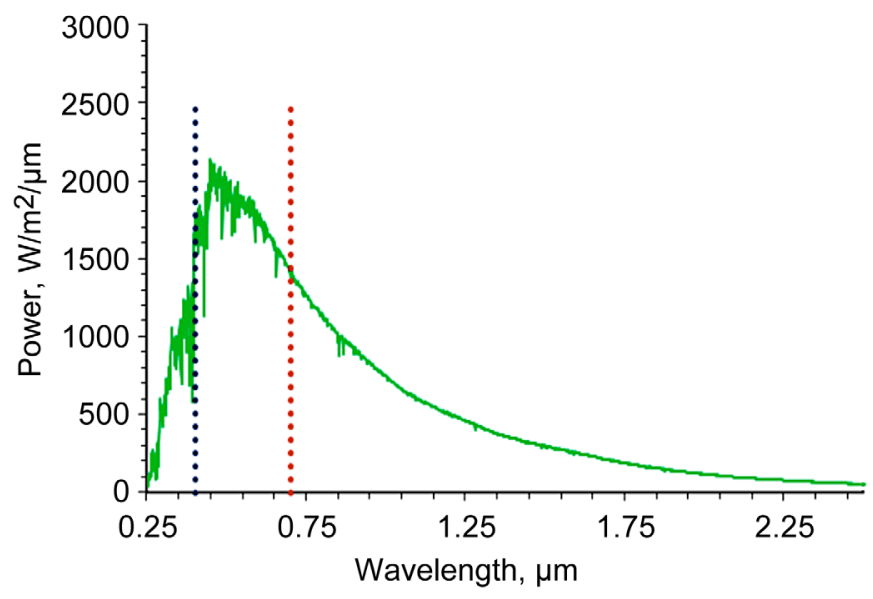

Figure 1. The solar spectrum measured at $1 \mathrm{AU}$ from the sun above the Earth's atmosphere (Air Mass Zero spectrum). ${ }^{2}$ The visible spectrum is the region between the dotted lines.

Table I. Temperatures of Interest and Their Corresponding Wavelengths According to the Raleigh-Jeans Law

\begin{tabular}{lcc}
\hline \multicolumn{1}{c}{ Feature } & Temperature, & Wavelength max, \\
& $\mathrm{K}$ & $\mu \mathrm{m}$ \\
\hline Permanently shadowed craters & 40 & 72 \\
Lunar night & 100 & 29 \\
Lunar polar regions & 200 & 14 \\
Lunar habitat temperatures & 300 & 10 \\
Lunar highest temperature & 400 & 7 \\
Nuclear reactor radiator & 1000 & 3 \\
$\quad$ temperature & & \\
\hline \hline
\end{tabular}


This preliminary report is being released due to the importance of getting simulant $\alpha$ data disseminated quickly. There are many researchers either working with these materials directly or trying to model results based on these materials for whom the thermal properties of $\alpha$ and $\varepsilon$ are key. As shown below, the $\alpha$ values were confounded by particle size effects. Future experiments will include regrinding and sieving the materials reported here to remove that variable. This report was written with the perspective of characterizing thermal characteristics rather than identifying structural features in underlying minerals, which will be reported in a future paper.

\section{Methods and Materials}

Eleven lunar simulants and one Mars simulant were included in this investigation. Four of the lunar simulants were fabricated independently, and the remaining seven were modifications on the basic four. The first of these basic four is JSC-1A, a mare-type simulant that is a remake of the JSC-1 simulant that has been the de facto NASA simulant since it was introduced in $1994 .{ }^{3}$ The second was a simulant developed by researchers at the University of Minnesota in 1988 approximating the bulk chemistry of Apollo 11 soil sample 10084, Minnesota Lunar Simulant \#1 (MLS-1). ${ }^{4}$ The third was developed in Japan in 1995 from the volcanic soils on Fujisan called FJS- $1 .^{5}$ The fourth was a lunar highlands type simulant produced through a current joint effort of NASA and the U.S. Geological Survey, NU-LHT. ${ }^{6}$

Three prototype JSC-1A lunar dust simulant variants were created by ORBITEC under a Phase I SBIR contract entitled "High-Fidelity Lunar Dust Simulant." The prototype lunar dust simulants were produced using a two-step process. In the first step, ORBITEC used a proprietary process to create a mature lunar regolith simulant that contains a high proportion of agglutinate-like particles and glass spherules, both of which contain metallic iron globules (including nanophase $\mathrm{Fe}^{0}$ ). This is the key innovation to creating a realistic lunar dust simulant since the majority of the lunar dust is composed of broken pieces of agglutinates. Samples of the processed JSC-1A simulants were sent to the NASA Glenn Research Center and the NASA Johnson Space Center for evaluation and characterization. The second step in making the prototype lunar dust simulants was an industrial milling process to grind the processed simulant materials down to the appropriate particle size distribution. Samples of the resulting prototype lunar dust simulants were also sent to the NASA Glenn Research Center and the NASA Johnson Space Center for evaluation and characterization.

The processed JSC-1A simulant (designated JSC-1A-5000-2X) contained numerous glass spherules 20 to $50 \mu \mathrm{m}$ in diameter, as well as more irregular glassy particles with admixtures of unmelted grains. Glass spherules with diameters as small as $5 \mu \mathrm{m}$ were observed. The JSC $-1 \mathrm{~A}-5000-2 \mathrm{X}$ material also contains sub-spherical glassy grains and glass shards. The glassy portions of the JSC $-1 \mathrm{~A}-5000-2 \mathrm{X}$ feedstock material contain numerous iron-rich globules in a range of sizes consistent with those found in lunar agglutinates, as seen within the limits of the scanning electron microscopes (SEM) at the NASA Glenn Research Center and the NASA Johnson Space Center. In the simulated agglutinate particles analyzed, the iron-rich globules range in size from less than $20 \mathrm{~nm}$ up to about $1 \mu \mathrm{m}$ in diameter. The same iron globules have been found extending into the glassy melt regions, though their size range is somewhat larger than found in regolith. ${ }^{7}$

Gas phase dispersal and aerosol diagnostic techniques were used by the NASA Glenn Research Center to measure the particle size distribution of the first two prototype dust simulants and JSC-1A lunar regolith simulant that was sieved to below $20 \mu \mathrm{m}$. Particles with aerodynamic diameters in the $\mu \mathrm{m}$ and $\mathrm{nm}$ range are routinely measured in the aerosol sciences (instruments can resolve particles with aerodynamic diameters down to 2.5 to $10 \mathrm{~nm}$ ). This technique does not require a priori assumptions about the particle size distribution function and is independent of refractive index and density. The same measurement technique was previously used to analyze the particle size distribution of two Apollo samples that were used to develop the prototype lunar dust simulants. ${ }^{8}$

Figure 2 shows the cumulative mass densities of the prototype dust simulants compared with JSC-1A (sieved below $20 \mu \mathrm{m}$ ) and two Apollo samples (10084 and 70051). The JSC-1A lunar regolith simulant and Apollo samples were all sieved below $20 \mu \mathrm{m}$. Both of the prototype lunar dust simulants are a better match to the Apollo particle size distribution than the original JSC-1A lunar regolith simulant. ${ }^{7}$

The JSC-1A-DUST-P6 was a sample of the prototype lunar dust simulant created by jet milling the JSC-1A5000-2X material to a particle size of less than $10 \mu \mathrm{m}$. The JSC-1A-AGGL-13000 was JSC-1A simulant that was sieved to remove particles greater than $1 \mathrm{~mm}$ and processed by ORBITEC to create simulated agglutinates. A slightly different processing method was used for this material, so it contained more glass and simulated agglutinates. The glass and simulated agglutinates probably contained metallic iron globules that ranged in size from $\sim 1 \mu \mathrm{m}$ down to the nm range, but the extensive analysis done for the JSC-1A-5000-2X material was not performed on this material. 


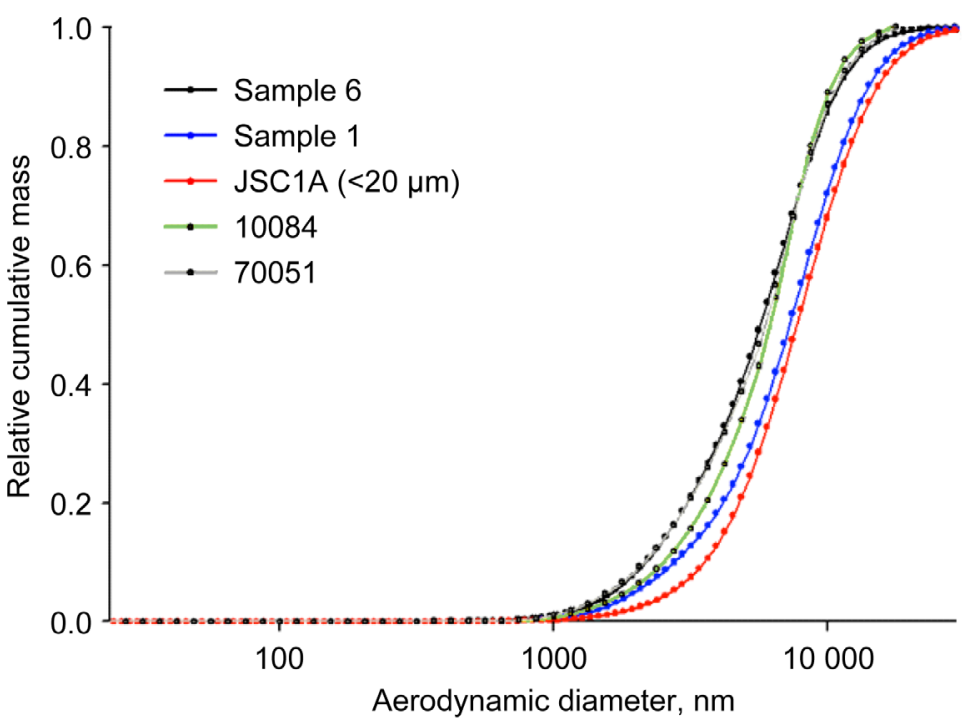

Figure 2. Cumulative mass densities of the prototype lunar dust simulants and Apollo samples from Greenberg et al. Sample \#6 in the legend refers to JSC-1A-DUST-P6. ${ }^{8}$

SEM backscattered electron images resolve droplet-shaped iron-rich globules that appear to be nanophase $\mathrm{Fe}^{0}$ on the surface of the larger glass particles in both of the first two prototype lunar dust simulants. The sub-micron grains appear to be covered with relatively large iron-rich droplets ( $\sim 25$ to $40 \mathrm{~nm})$ and very small droplets ( $\sim 5$ to $10 \mathrm{~nm})$. Scanning transmission electron microscope (STEM) examination found silicate glass that contains numerous nanophase grains. Energy dispersive spectroscopy analyses indicate that the nanophase grains are primarily metallic iron $\left(\mathrm{Fe}^{0}\right)$ mixed with some iron oxide. Transmission electron microscopy (TEM) analyses were also performed on the prototype dust simulants to investigate the presence of nanophase $\mathrm{Fe}^{0}$. The observation of iron-rich globules smaller than $20 \mathrm{~nm}$ in the lunar dust simulant grains is very similar to previous observations of iron-rich globules in simulated agglutinate particles. The most likely form of the iron-rich globules is metallic iron $\left(\mathrm{Fe}^{0}\right)$ located on and within glassy grains, but the exact composition of the iron-rich particles has not yet been determined due to the extremely small particle sizes. Attempts to measure the electron diffraction pattern of the iron-rich particles caused the grains to melt. ${ }^{7}$

The MLS-1P variant was produced by dropping the MLS-1 through a $6000{ }^{\circ} \mathrm{C}$ plasma in an in-flight sustained shockwave plasma reactor, $10 \mathrm{~m}$ tall. Glass content from 10 to 30 percent was achieved with feed rates of 40 to $50 \mathrm{~kg} / \mathrm{hr}$. Although no agglutinate-like materials were produced, part of the simulant was melted into glassy spheroids, some as large a $2 \mathrm{~mm}$.

Two generations of NU-LHT simulant were tested. NU-LHT-1 contains principally plagioclase, which dominate the highland regions. The sample tested included only the dust fraction, (NU-LHT-1D) the result of sieving to remove particles larger than $30 \mu \mathrm{m}$. NU-LHT-2 is similar NU-LHT-1 to which minor amounts of olivine, whitlockite, and fluorapatite were added, and harzburgite removed. In addition, the relative amount of norite was decreased and agglutinates increased to more closely match the regolith. NU-LHT-2M was the result of sieving to remove particles larger than $500 \mu \mathrm{m}$.

Two additional variants were made from the NU-LHT-2M. The first was processed by ORBITEC to create glass and simulated agglutinates using the process described for JSC-1 A above and was designated NU-LHT-2M700-1X. Although the presence of simulated agglutinates with iron globules was confirmed, no analysis to further characterize this material had been completed at the time of this writing. Part of this material was then jet milled to reduce its particle size and was designated NU-LHT-700-1X.

Although the focus of this study was lunar soil simulants, the Martian soil simulant JSC Mars-1 was included for comparison. This simulant was collected from the Pu'u Nene cinder cone in Hawaii and was developed to be a spectral match to the bright regions of Mars. ${ }^{9}$ It is commercially available from ORBITEC. Table II summarizes important characteristics of the simulants used in this study.

The reflectance spectra in the 250 to $2500 \mathrm{~nm}$ range were collected on a Cary 5000 spectrophotometer (Varian) equipped with a DRA $2500,150 \mathrm{~mm}$ diameter integrating sphere. A Varian powder attachment was filled with 10 grams of sample which resulted in a sample 4 to $5 \mathrm{~mm}$ thick, depending on how finely the simulant was ground. 
Table II. Simulants Used in This Study

\begin{tabular}{lccc}
\hline \hline \multicolumn{1}{c}{ Simulant } & Region & Particle Size* & Agglutinates \\
\hline JSC-1AF & mare & small & no \\
JSC-1A-5000-2X & mare & large & yes \\
JSC-1A-DUST-P6 & mare & small & yes \\
JSC-1A-AGGL-13000 & mare & large & yes \\
FJS-1 & mare & large & no \\
MLS-1 & mare & large & no \\
MLS-1P & mare & large & glass \\
NU-LHT-1D & highlands & small & no \\
NU-LHT-2M & highlands & small & no \\
NU-LHT-2M-700 & highlands & large & yes \\
NU-LHT-700 & highlands & small & yes \\
JSC Mars-1 & Mars & large & no \\
\hline \hline
\end{tabular}

*Simulants with "large" particle sizes exhibited even flow, while those with

"small" particles sizes did not.

A second, manufacture-provided powder attachment filled with powdered Spectralon ${ }^{\circledR}$, the same material that coated the inside of the integrating sphere, was used for the baseline. Light entered the cells through matching quartz windows. Data were collected from 250 to $2500 \mathrm{~nm}$ in increments of $1 \mathrm{~nm}$, at a scan rate of $600 \mathrm{~nm} / \mathrm{min}$. A deuterium lamp was used to measure the 250 to $350 \mathrm{~nm}$ data, and a halogen lamp to measure the 350 to $2500 \mathrm{~nm}$ data. Immediately prior to running each sample, a spectrum of the Spectralon ${ }^{B}$ was collected as a sample, to determine whether the baseline was still valid. In all instances the deviations in the baseline were less than 1 percent. Three spectra of each sample were collected consecutively. The sample holder was rotated between each spectrum so that the beam sampled a different area of the sample. The three spectra were averaged, point by point, and multiplied by the ratio of blank to baseline. A set of eight NIST traceable diffuse reflectance standards (Labsphere) with $\rho$ ranging from 0.02 to 0.99 was measured through the quartz window as well. A correction factor was applied to the averaged $\rho$ for each sample to place it on an absolute scale. These data were used in the analysis.

With a sample thickness of $4 \mathrm{~mm}$ and a black backing plate, it was assumed that there was no transmission through the sample. Under these conditions $\alpha(\lambda)=(1-\rho(\lambda))$. The solar $\alpha$ of each sample was determined by calculating the convolution of the simulant with the ASTM air mass zero solar spectrum E-490-00 and expressed as a fraction of the solar spectrum. An example of the convolution with JSC-1AF superimposed on the AM0 solar spectrum is shown in Fig. 3.

Reflectance spectra in the 2,500 to $25,000 \mathrm{~nm}$ range were collected on a Magna-IR 760 (Nicolet) equipped with a Labsphere RSA-NI-550ID $76 \mathrm{~mm}$ Infragold $^{\circledR}$ integrating sphere using a deuterated triglycidyl sulfate detector. This is a Fourier transform instrument and 100 scans with a data point spacing of $7.714 \mathrm{~cm}^{-1}$ were collected for each sample. A gold background spectrum was measured prior to each scan. The integrating sphere was purged with nitrogen to eliminate background water and carbon dioxide bands. Powder samples of 4 to $6 \mathrm{~mm}$ thickness were place in a Fluoroware dish which was placed at the sample port at the bottom of the integrating sphere. No window was necessary in this configuration. None of the Fluoroware spectral peaks were visible in any of the spectra, indicating that the samples were optically opaque. During the

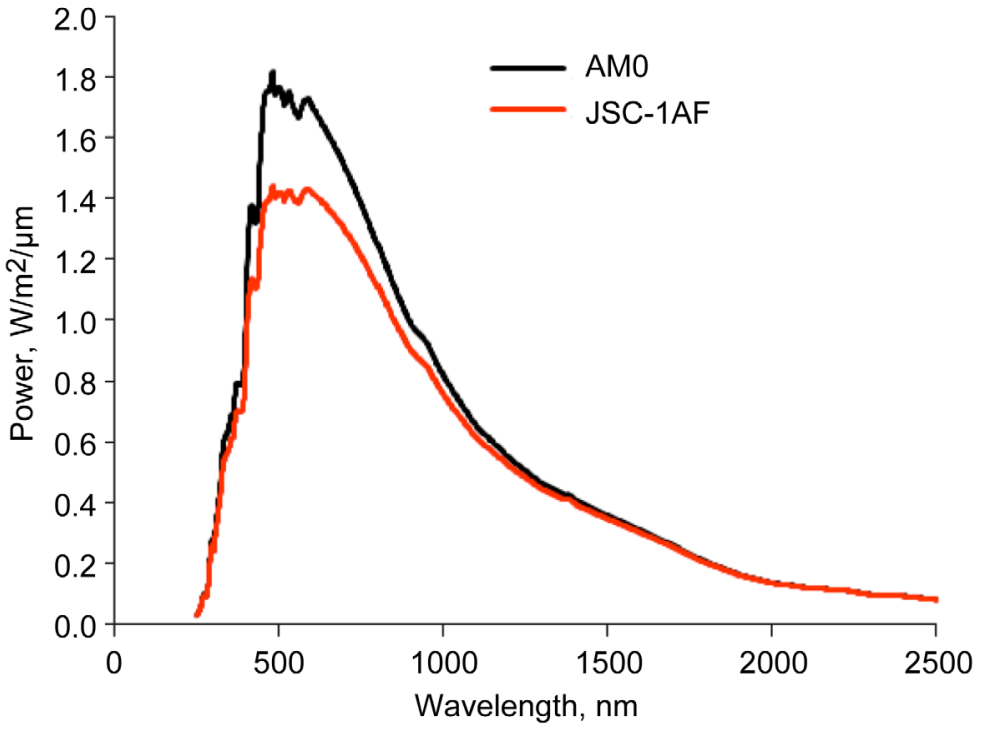

Figure 3. The air mass zero solar spectrum and its convolution with the JSC-1AF spectrum. The area under the JSC-1AF curve is the areal power absorbed by the simulant. 
course of the data collection two NIST traceable standards of similar reflectivity (SRS-10-010 and SRS-20-010) were measured as well.

The $\varepsilon$ was determined over the full temperature range expected on the lunar surface, 100 to $400 \mathrm{~K}$. This was calculated by convoluting the 2.5 to $25 \mu \mathrm{m}$ wavelength $\rho(\lambda)$ data with blackbody curves over the temperature range in $5 \mathrm{~K}$ increments. ${ }^{10}$ The full range of the $\varepsilon$ over that temperature span was only a few percent for all of the samples, except the MLS-1. Only the $\varepsilon$ at $300 \mathrm{~K}$ is reported here.

There are subtle differences between the ways the data were collected on the two spectrophotometers. In the Cary, the sample was physically substituted for the reference on the surface of the integrating sphere. But in the Nicolet, the sample was measured by alternating a mirror between positions where the reference was measured, or the sample was measured. So in the case of the Nicolet, some small fraction of the integrating sphere when the reference is being measured is covered with the sample, whereas in the Cary it is not. This correction factor is dependant on the reflectance of the sample. Both instruments measured the samples at $2500 \mathrm{~nm}$, so this was used to scale the Nicolet data to the Cary data. A plot of the Cary data as a function of the Nicolet data revealed that the $\rho(\lambda)$ varied linearly with an $\mathrm{R}^{2}$ of 0.97 . This correction factor was applied to all of the Nicolet data. Deviations between the calculated and experimental data at $2500 \mathrm{~nm}$ appear as a small discontinuity in the $\rho(\lambda)$ plots below.

\section{Results and Discussion}

Particle size is known to affect the $\rho(\lambda)$ of powdered rock and mineral samples. This was dramatically shown in the case of lunar regolith in the data of Taylor et al., who measured the $\rho(\lambda)$ of sieved fractions of lunar samples returned during Apollo. ${ }^{11}$ They found that smaller particles were both more reflective and showed fewer spectral features. The specimens measured in the present study were not of uniform particle size distribution, nor even of the same mean size. There was a large variation in the particle sizes of the simulants in this study, and they were lumped into large or small particle sizes depending on whether they flowed freely from the storage bottles to the Cary powder measurement attachment. Those listed in Table II as "small" packed into the attachment tightly enough to have appreciable cohesion, forming pellets under the pressure of the powder attachment.

The reflectance spectra of JSC-1AF, NU-LHT-1D, MLS-1, FJS-1, and JSC Mars -1 are shown in Fig. 4. Particle size effects skew the absolute value of the $\rho(\lambda)$, with the smaller particle sizes having higher $\rho$. Even without taking particle size into account, it is clear that there is little difference in the $\rho(\lambda)$ at wavelengths greater than about $8000 \mathrm{~nm}$, except for the case of MLS -1 . Further, the infrared $\rho(\lambda)$ for those simulants is about 0.10 for wavelengths longer than $8000 \mathrm{~nm}$. Using
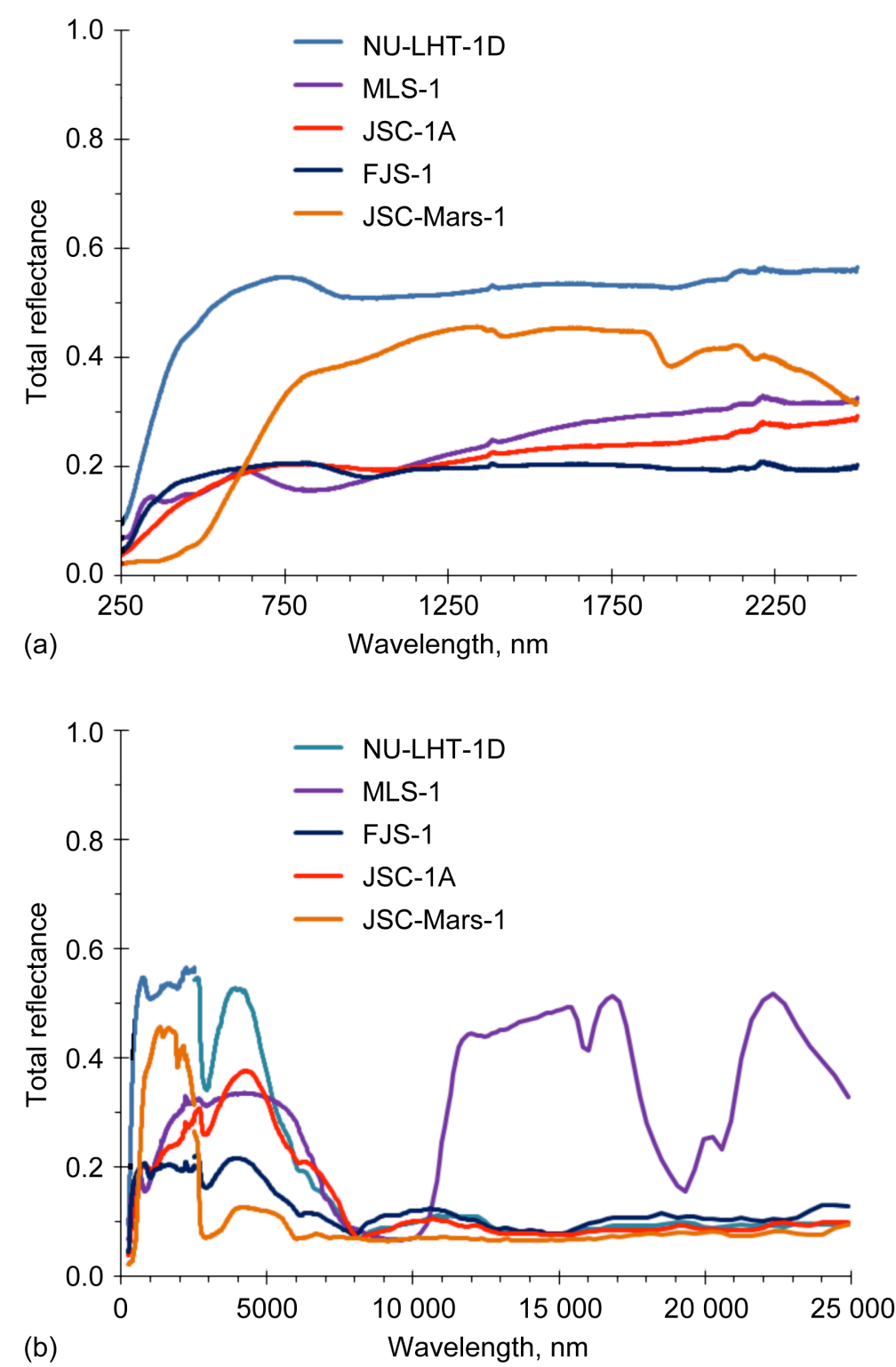

Figure 4. The reflectance spectra of the four lunar simulants and the Mars simulant over the absorptive (a) and full (b) spectral ranges. 
Table III. The Solar $\alpha$ and Thermal $\varepsilon$ Calculated for Simulants Tested Having Small Particle Sizes

\begin{tabular}{lccc}
\hline \multicolumn{1}{c}{ Simulant region } & $\alpha$ & $\varepsilon(300 \mathrm{~K})$ & \\
\hline NU-LHT-700 & highlands & 0.413 & 0.902 \\
NU-LHT-1D & highlands & 0.444 & 0.891 \\
NU-LHT-2M & highlands & 0.556 & 0.884 \\
JSC-1A-DUST-P6 & mare & 0.642 & 0.899 \\
NULHT-JSC1A & mixed & 0.662 & 0.888 \\
JSC-1AF & mare & 0.817 & 0.895 \\
\hline \hline
\end{tabular}

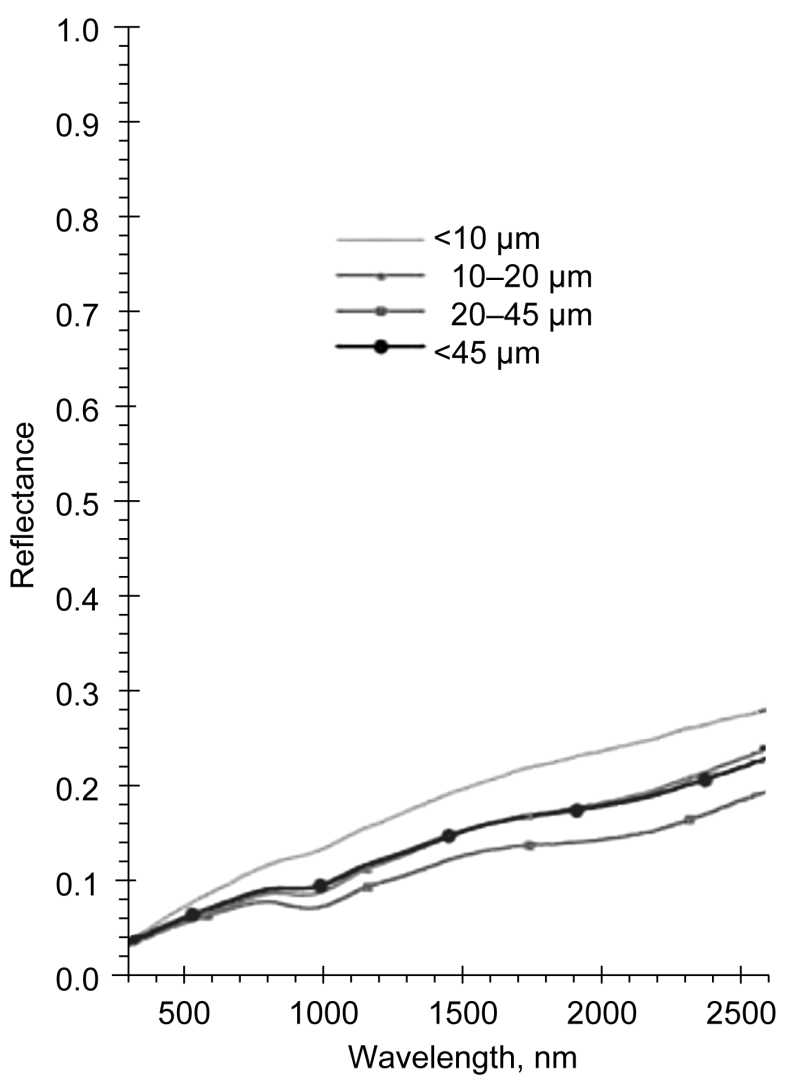

Table IV. The Solar $\alpha$ and Thermal $\varepsilon$ Calculated for Simulants Tested Having Large Particle Sizes

\begin{tabular}{lccc}
\hline \multicolumn{1}{c}{ Simulant region } & $\alpha$ & $\alpha(300 \mathrm{~K})$ \\
\hline NU-LHT-2M-700 & highlands & 0.669 & 0.901 \\
JSC Mars-1 & Mars & 0.743 & 0.927 \\
FJS-1 & mare & 0.812 & 0.895 \\
MLS-1 & mare & 0.816 & 0.704 \\
MLS-1P & mare & 0.850 & 0.895 \\
JSC-1A-AGGL-13000 & mare & 0.907 & 0.905 \\
JSC-1A-5000-2X & mare & 0.923 & 0.906 \\
\hline \hline
\end{tabular}

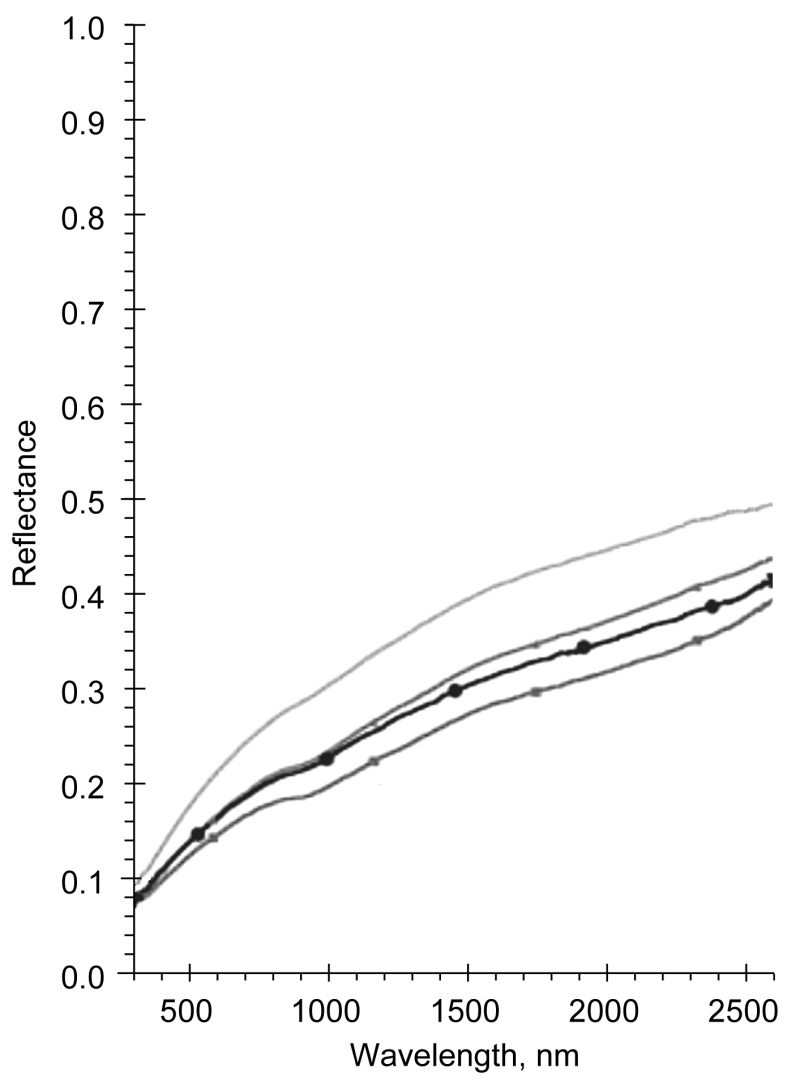

Figure 5. The bidirectional reflectance spectra (a) Apollo 12001 mare and (b) Apollo 64801 highland lunar soils. $^{13}$

the Raleigh-Jeans law this corresponds to about $360 \mathrm{~K}$, so for the temperatures at which the thermal control surfaces are expected to operate under lunar conditions, the $\varepsilon$ 's are all about the same. This implies that the thermal emission properties all over the lunar surface are likely to be similar, and in the range of 0.9 . The $\varepsilon$ at $300 \mathrm{~K}$ for each of the simulants is given in Tables III and IV. If MLS-1 is excluded, the lunar simulants $\varepsilon$ 's range from 0.884 to 0.906 with an estimated error of \pm 0.005 . Note that particle size does not appear to have a large effect on the $\varepsilon$ 's of the simulants. It is interesting that the value of the Martian simulant is somewhat higher, 0.927.

Figure 5 shows bidirectional reflectance spectra measured of a mare soil (12001) and a highlands soil (64801) returned by Apollo 12 and 16, respectively. ${ }^{12}$ Note that, as seen in the samples in this study, the $\rho(\lambda)$ increases as the particle size decreases. Pieters and Taylor attribute this, at least in part, to their observation that the finer fraction contains more feldspar, which is lighter in color than the more mafic pyroxenes. The composition of the simulants, however, is nearly invariant between the larger and smaller components because the fine fractions are produced from grinding the coarser fractions. Unlike the lunar simulants in Fig. 4(a), the $\rho(\lambda)$ of lunar soils increases almost monotonically over the range from 300 to $2500 \mathrm{~nm}$. This is often attributed to agglutinitic glass, and the nanophase iron it contains. However, this trend in the $\rho(\lambda)$ was not observed in simulants with agglutinitic glasses added. 
Finally, the finest fraction of the mare soil has an $\alpha \approx 0.82$, and the coarsest fraction about 0.88 , well within the ranges of the mare simulants tested here (Tables III and IV). The $\alpha$ of the highland soils varies from about 0.65 for the finest fraction to about 0.73 for the coarsest fraction, again within the range of the simulants tested. So although there are some spectral differences, the total integrated $\alpha$ for the simulants appears to be typical for lunar soils.

Even though they are similar in the long wavelength region, there are large differences among the five basic simulants in the $\rho(\lambda)$ in the 250 to $2500 \mathrm{~nm}$ range, where the solar spectrum is the most intense. NU-LHT-1D, the lone lunar highlands simulant of the five, is much more reflective in the wavelength region below $5000 \mathrm{~nm}$ than the others. Although the JSC -1 AF and MLS -1 samples had very similar values of $\rho(\lambda)$ in this region, MLS -1 has much larger particle size, leading to the conclusion that JSC-1AF is probably an intrinsically darker material. While FJS-1 appears to be the darkest of the lunar simulants, its large particle size darkens it such that the intrinsic $\rho$ may not be darker than JSC-1AF. The lone Martian simulant has a high $\rho(\lambda)$ in the 250 to $2500 \mathrm{~nm}$ wavelength region in spite of the fact that the particle size was very large, greater than $1000 \mu \mathrm{m}$. The Martian soil simulant probably has the highest intrinsic $\rho$, as would be expected from the higher albedo of the Martian surface, compared to the lunar surface. Even before a quantitative analysis, it is apparent from these spectra that there will be substantial variations in the solar $\alpha$ among the simulants.

MLS-1 is much more reflecting than the others in the 10 to $25 \mu \mathrm{m}$ wavelength range. Since MLS-1P is much more absorbing in this range (Fig. 6), it is thought that the difference is primarily due to glass content. MLS-1 contains no glass or agglutinates. ${ }^{13}$ Glasses are known to dramatically increase absorption over crystalline materials in this portion of the spectrum. In addition, the other simulants all have high glass content, as does the lunar soil. So MLS-1 would probably not be a good simulant to use if the $\varepsilon$ properties are important to the test.

Figure 7 shows $\rho(\lambda)$ for JSC-1AF and its variants. The addition of agglutinates in the JSC $-1 \mathrm{~A}-5000-2 \mathrm{X}$ and JSC-1A-AGGL-13000 variants has little effect on the reflectivity at wavelengths greater than $8000 \mathrm{~nm}$, but there is a darkening at 250 to $8000 \mathrm{~nm}$ which is similar for both. Once again particles size effects are important here, as the agglutinated samples have considerably larger particle size. This is illustrated by comparison of the JSC-1A-5000-2X to its milled variant, JSC-1A-DUST-P6 where the latter variant is considerably lighter in the 250 to $8000 \mathrm{~nm}$ range.

Figure 8 shows $\rho(\lambda)$ for NU-LHT and its variants. As related above, the particle size is probably the biggest difference between NU-LHT-1D and NU-LHT-2M. It can be seen that the $\rho(\lambda)$ in the 250 to $2500 \mathrm{~nm}$ range is somewhat higher for the smaller NU-LHT-1D, and at the longer wavelengths the $\rho(\lambda)$ of the two are comparable. The addition of the agglutinates in NU-LHT-2M-700 darkens the $\rho(\lambda)$ in the 250 to $2500 \mathrm{~nm}$ range, but upon grinding (NU-LHT-700) the $\rho(\lambda)$ increases in that region to where it has a higher $\rho(\lambda)$ than even NU-LHT-1D. NU-LHT-700 is the darkest of the NU-LHT variants at wavelengths greater than $8000 \mathrm{~nm}$, indicating that glass content is more important than particle size in the thermal infrared.

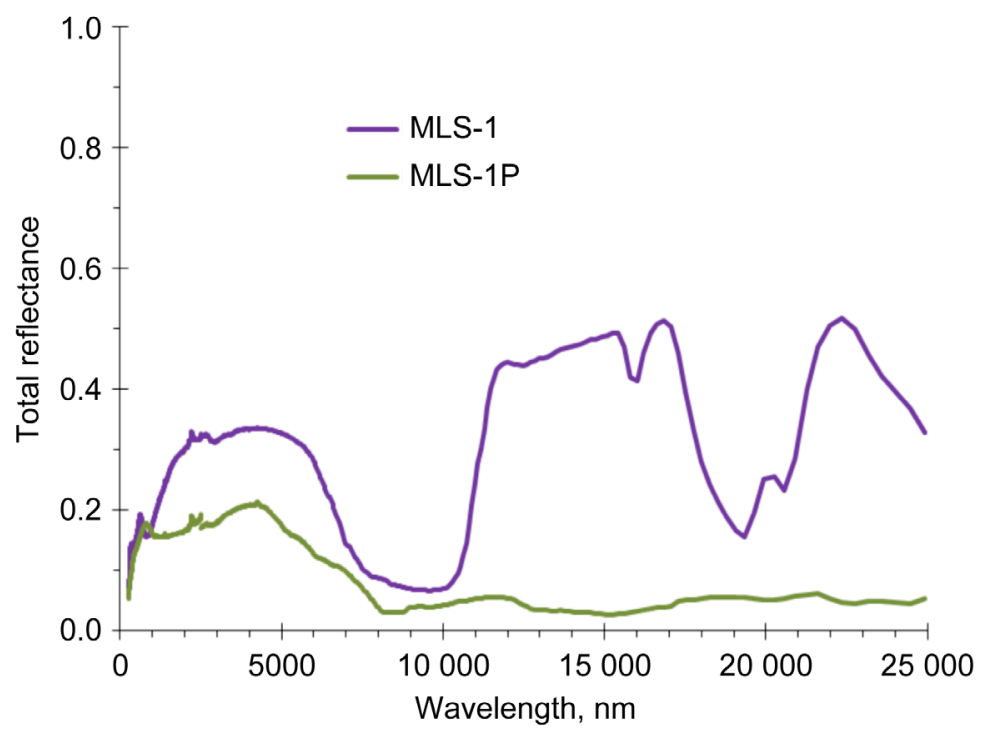

Figure 6. The reflectance spectra of the MLS-1 and MLS-1P lunar simulants. 


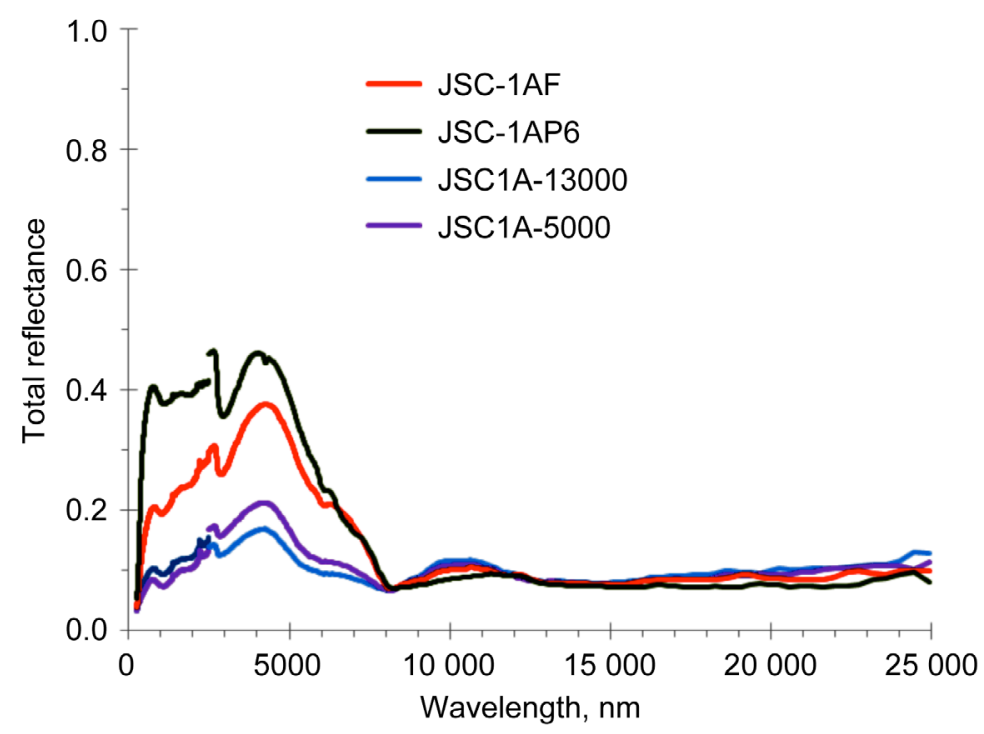

Figure 7. The reflectance spectra of the four variants of JSC-1A lunar simulants.

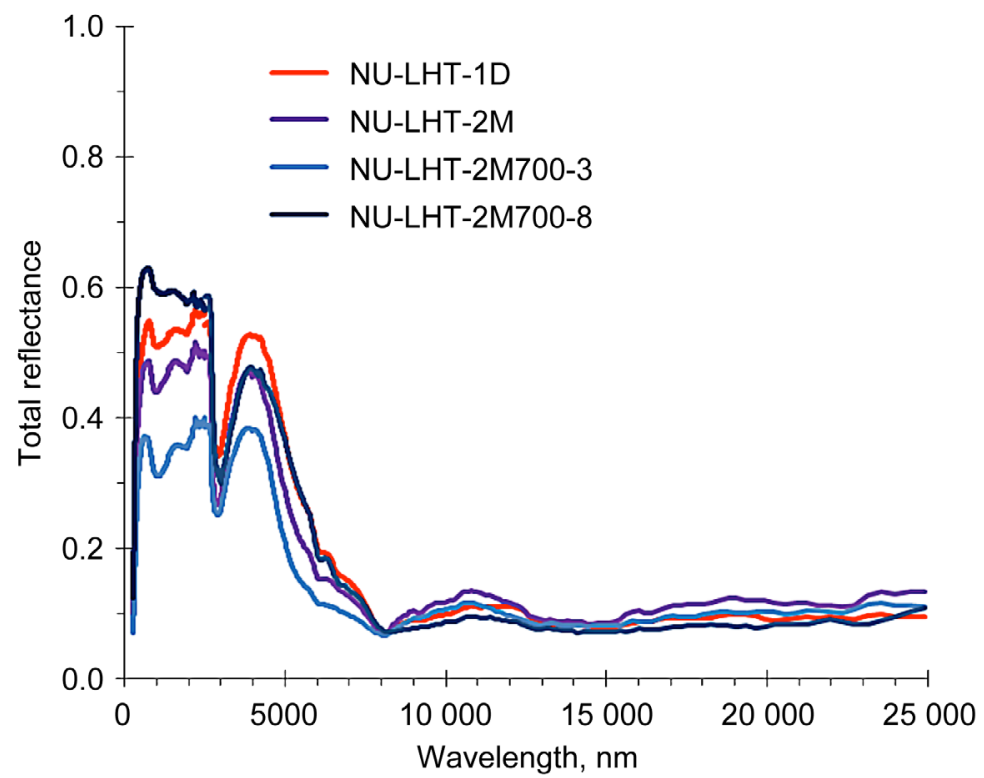

Figure 8. The reflectance spectra of the four variants of NU-LHT lunar simulant.

Like most lunar soils, the lunar simulants fall into two broad categories, the darker mare simulants and the lighter highlands simulants. In order to determine whether the effects on thermal control systems varies linearly with the darkness of the soil, a simulant of intermediate $\alpha$ was desired. To accomplish this a 1:1 mixture of the light highlands simulant NU-LHT-1D and the dark mare simulant JSC-1AF was prepared. This also provided an opportunity to see how additive the $\alpha$ and $\varepsilon$ of these multicomponent mixtures are. The results are shown in Fig. 9, which shows the mixed simulant spectrum along with those of its constituents. Also shown is a point by point average of the two. It can be seen that the point-by-point average lies nearly on top of the 1:1 mixture, showing that the $\rho(\lambda)$ is additive. 


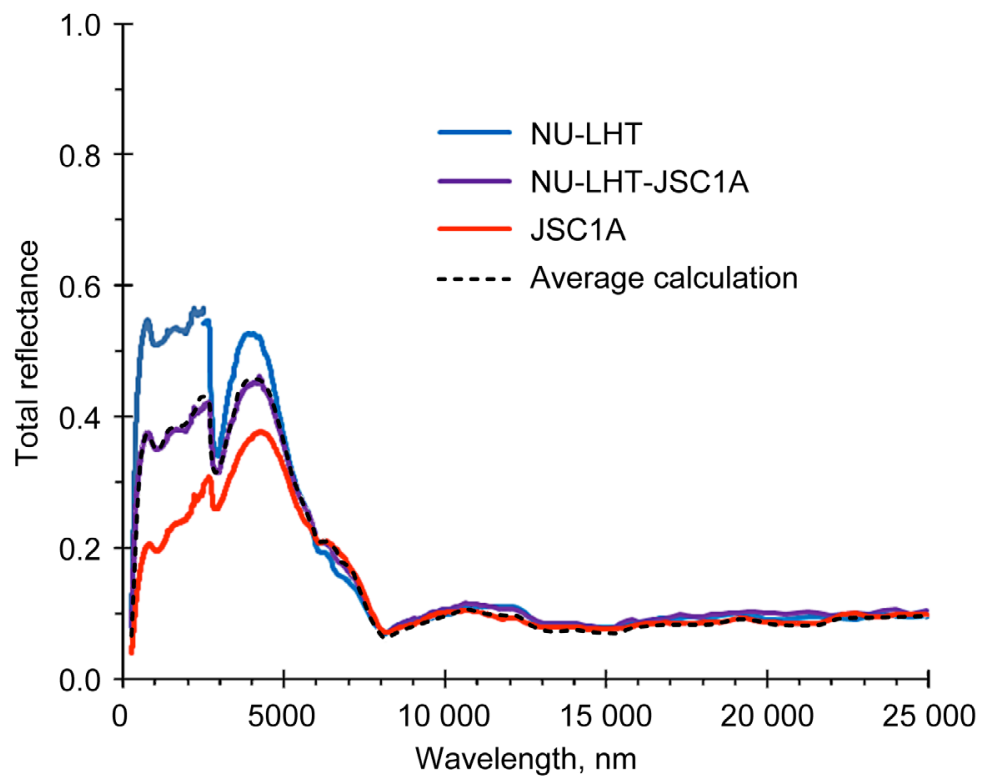

Figure 9. The reflectance spectra of JSC-1AF, NU-LHT-1D and a 1:1 mixture of the two. The dotted line shows the point by point average of JSC-1AF and NU-LHT-1D.

\section{Conclusions}

The first comparative study of the reflectance spectra of lunar simulants was presented. Direct quantitative comparisons were hampered by the fact that there was a considerable range of particle sizes among the samples, and the reflectance of larger particles is less than smaller. All of the simulants except MLS-1 had $\rho(\lambda)$ of about 0.1 over the wavelength range of 8 to $25 \mu \mathrm{m}$, so they are very absorbing in the room temperature and lower. This implies that they are also highly emitting at room temperature and lower. An indication of this is that the $\varepsilon$ at $300 \mathrm{~K}$ of all the lunar simulants except MLS-1 ranged from 0.884 to 0.906 . MLS-1, which has essentially no glass content, was quite reflective in the thermal infrared region with a $300 \mathrm{~K} \varepsilon$ of 0.704 , well below that reported for lunar soils. Thus, MLS -1 is not recommended for any application for which the $\varepsilon$ of the simulant would be important. The $300 \mathrm{~K} \varepsilon$ of JSC Mars-1 simulant was appreciably higher than any of the lunar simulants (0.927).

There was considerably more variation in the lunar simulant reflectance in the solar spectral range (250 to $2500 \mathrm{~nm}$ ) than in the thermal infrared. Larger particle size simulants reflected much less than those with smaller particle size. As expected, the lunar highlands simulants were more reflective in this wavelength range than the lunar mare simulants. The $\alpha$ of the simulants ranged from 0.413 to 0.817 for those with smaller particles and 0.669 to 0.906 for large particles. Although the ranges of $\alpha$ were similar to that measured for lunar mare and highland soils ( 0.65 to 0.88 ), this is considerably less than the values often used in thermal modeling of the lunar surface.

Thermal models are often based on $\rho$ 's derived from albedo measurements. Any albedo measurements made from orbit or earth will necessarily include particles from sub-micron dust up to boulders many meters across. This will also include areas that are shadowed which will appear to darken the albedo. Although these measurements will be useful to model the overall thermal environment of the lunar surface, they will not be as useful in predicting how collateral dust and soil will affect thermal control systems. The collateral dust will be dominated by small particles, and partially successful cleaning efforts will leave even a smaller size fraction. This study shows that the extent to which the dust will affect thermal control systems depends on whether they are operating in a mare region, where the soils are relatively dark, or in a highlands region, where the soils are relatively light.

\section{Acknowledgements}

The authors gladly acknowledge the contributions of D. Rickman of the NASA Marshall Spaceflight Center, and D. Stoesser and S. Wilson of the U.S. Geological Survey for their many helpful discussions about the properties of the lunar regolith and lunar simulants. In addition, S.K.R. Miller and D.A. Jaworske of the NASA Glenn Research Center freely shared their guidance and expertise in the spectral measurements. Programmatic and 
financial support from the NASA Exploration Technology Development Program came through the Dust Mitigation Project (M. Hyatt at the NASA Glenn Research Center) and the Advanced Thermal Control Project (R. Stephan, NASA Johnson Space Center.)

\section{References}

${ }^{1}$ NASA SP-315, “Apollo 16 Preliminary Science Report," Chapters 6 and 19, (NASA, 1972).

${ }^{2} 2000$ ASTM Standard Extraterrestrial Spectrum Reference E-490-00, http://rredc.nrel.gov/solar/spectra/AM0.

${ }^{3}$ McKay, D.S. et al., "JSC-1: "A New Lunar Soil Simulant," Engineering, Construction, and Operations in Space IV, American Society of Civil Engineers, 1994, pp. 857-866.

${ }^{4}$ Weiblen, P.W., and Gordon, K., "Characteristics of a Simulant for Lunar Surface Materials," Second Conference on Lunar Bases and Space Activities in the $21^{\text {st }}$ Century, LPI Contribution 652.

${ }^{5}$ Kanamori, H. et al., "Properties of Lunar Soil Simulant Manufactured in Japan," Proceedings of the $6^{\text {th }}$ International Conference and Exposition on Engineering, Construction, and Operations in Space, ASCE, Albuquerque, NM (1998).

${ }^{6}$ Stoeser, D.B. et al., "Development of Lunar Highland Type Simulants, NU-LHT-1M, -2M," Geochemica et Cosmochemica Acta, Vol 72, No. 12, pA902.

${ }^{7}$ Gustafson, R.J., White, B.C., 2009. "Development of a Lunar Dust Simulant," International Conference of Environmental Systems (ICES) Proceedings: 09ICES-0125. Society of Automotive Engineers (SAE), Warrendale, PA.

${ }^{8}$ Greenberg, P., Chen, D., Smith, S., 2007. "Aerosol Measurements of the Fine and Ultrafine Particle Content of Lunar Regolith,” NASA/TM-2007-214956.

${ }^{9}$ Allen, C.C. et al., "JSC Mars-1: Martian Regolith Simulants," Lunar and Planetary Science XXVIII, 1997, 1797.pdf.

${ }^{10}$ Jaworske, D.A., and Skowronski, T.J., "Portable Infrared Reflectometer for Evaluating Emittance," Space Technology and Applications Forum - 2000, Ed., M.S. El-Genk. American Institute of Physics, 2000, CP504.

${ }^{11}$ Taylor, L.A. et al., "Lunar Mare Soils: Space Weathering and the Major Effects of Surface-Correlated Nanophase Fe," J. Geophys Res. Vol 106 No. E11, 2001, pp. 27985-27999.

${ }^{12}$ Pieters, C.M., and Taylor, L.A., "Systematic Global Mixing and Melting in Lunar Soil Evolution," Geophys. Res. Let, Vol. 30, No. 20, 2048, doi10.1029/23GLO18212, 2003.

${ }^{13}$ Bariste, S.N., and Sture, S., "Lunar Regolith Simulant MLS-1: Production and Engineering Properties," Lunar Regolith Materials Workshop, NASA Marshall Space Flight Center, Jan 24-26, 2005, pp. 12-13. 